\title{
Importance of Pre-Anesthetic Evaluation: Case Report of a Patient with Obstructive Sleep Apnea
}

\author{
Raquel Reis Soares, TSA ${ }^{1}$, Friederike Wolff Valadares ${ }^{2}$, Marta Beatriz Araujo ${ }^{2}$, Michele Nacur Lorentz, TSA ${ }^{3}$
}

Summary: Soares RR, Valadares FW, Araujo MB, Lorentz MN - Importance of Pre-Anesthetic Evaluation: Case Report of a Patient with Obstructive Sleep Apnea.

Background and objectives: The objective of this report was to show the importance of pre-anesthetic assessment in reducing morbidity and mortality of surgical patients and alert to problems and anesthetic care associated with obstructive sleep apnea.

Case report: This is a 28-year old male patient with class II obesity, sleep apnea, hypertension, referred to the pre-anesthetic outpatient clinic for evaluation of uvuloplasty under general anesthesia. After orientation on sleep apnea, explaining to the patient the risks associated with the procedure, and discussing with the surgeon, we decided to review the surgical indication and postpone the procedure.

Conclusions: The pre-anesthetic outpatient clinic is extremely important in reducing morbidity as well as mortality of surgical patients and, especially, in evaluating patients with obstructive sleep apnea, showing to be fundamental for the anesthetic-surgical schedule, orienting conducts to be followed, and interfering positively on patient prognosis.

Keywords: Anesthesia, General; Sleep Apnea, Obstructive; Analgesics, Opioids.

๑2011 Elsevier Editora Ltda. All rights reserved.

\section{INTRODUCTION}

The pre-anesthetic outpatient clinic should be used by all anesthesiology services for preoperative evaluation of patients, increasing the safety of anesthesia, elucidating patients, reducing the number of surgeries cancelled, and increasing patient satisfaction.

Obstructive sleep apnea (OSA) is characterized by a partial periodic obstruction of the airways during sleep. Obstruction leads to frequent awakening to recover the permeability of the airways. The poor quality of sleep interrupted at night favors daytime somnolence and other manifestations of sleep deprivation, such as memory loss, worsening of motor coordination, fatigue, irritability, reduced concentration, decline of cognitive function, decrease in performance, and reduction of physical well-being and quality of life ${ }^{1}$.

During sleep, oxygen desaturation and hypercarbia associated with cardiovascular changes of OSA episodes can be observed. A recent study published by the Chicago University

\footnotetext{
Received from Hospital Biocor Nova Lima MG, Brasil

1. Anesthesiologist; Pain Specialist of the Brazilian Society for Pain Study (SBED)

2. Anesthesiologist at Hospital Biocor - Nova Lima, MG

3. Professor of Anesthesiology, Universidade Federal de Minas Gerais (UFMG); Anesthesiologist at Hospital Biocor
}

Submitted on November 7, 2010

Approved on February 21, 2011.

Correspondence to:

Dra. Raquel $R$ Soares

Rua Elza Brandão Rodarte, 330/600

30320630 - Belo Horizonte, MG, Brazil.

E-mail: raquelrsoares@globo.com demonstrated the relationship between sleep deprivation and glucose intolerance and cardiovascular diseases ${ }^{2}$. The stress generated by insufficient sleep both in quality and quantity increases cortisol, adrenaline, and noradrenaline secretion, resulting in insulin resistance with predisposition for type II diabetes, cardiovascular diseases such as hypertension, dysrhythmias, myocardial infarction, stroke, and chronic pain. Therefore, adequate sleep in the treatment of patients with chronic pain is fundamental. Insomnia reduces the immunologic response and leptin levels (anorectic hormone produced by adipocytes), increasing hunger and stimulating appetite ${ }^{3,4}$. Treatment of sleep disorders has a chance to improve glucose metabolism and energetic balance 5 .

It has been estimated that the prevalence of obstructive respiratory disorders in adults measured in sleep laboratories is $9 \%$ in female and $24 \%$ in male, and it is closely related to obesity. Classic obstructive apnea on the other hand has an incidence of $2 \%$ in female and $4 \%$ in male, increasing in the elderly and more obese patients ${ }^{6}$. Approximately $85 \%$ of OSA cases are not diagnosed, and life expectancy of these patients is well below that of the general population 7,8 .

In the perioperative period, this type of patient represents a challenge to the anesthesiologist and should receive an adequate approach to reduce perioperative morbidity and mortality risks, as well as to improve patient prognosis ${ }^{9}$.

\section{CASE REPORT}

A male patient, 28 years old, with diagnosis of grade II obesity was referred to the pre-anesthetic outpatient clinic of the Hos- 
pital Biocor in Nova Lima, MG, Brazil, by the ENT for surgery for snoring: uvuloplasty.

The patient had a history of mild hypertension, asthma, daily use of bronchodilator, heavy smoker, with glucose intolerance. He reported loud snoring and daytime fatigue.

Preoperative exams showed: hematocrit $46 \%$; hemoglobin $16 \mathrm{mg} \cdot \mathrm{dL}^{-1}$; creatinine $0.96 \mathrm{mg} \cdot \mathrm{dL}^{-1}$; and glucose $102 \mathrm{mg}^{\mathrm{dL}}{ }^{-1}$. Echocardiogram showed preserved left ventricular function, and stress test showed low inotropic response without any other changes. He also had a polysomnography, which indicated the use of continuous positive airway pressure (CPAP). A cardiology evaluation classified the patient as ASA II.

On physical examination, the patient weighed $130 \mathrm{~kg}$, height $180 \mathrm{~cm}$, body mass index (BMI) 38, had good general status, mucous membrane pink, hydrated, with good peripheral perfusion. Cardiovascular assessment revealed BP $140 / 90 \mathrm{mmHg}$, heart rate 85 beats per minute, and fixed splitting of the second heart sound. Respiratory auscultation showed clear sounds with bibasilar reduction of breath sounds. Mallampati testing classified as 4 , thyromental distance $7 \mathrm{~cm}$, and neck circumference $44 \mathrm{~cm}$. The anesthesiologist reclassified the patient as ASA III because, besides obesity, mild hypertension, and asthma controlled with bronchodilators, the patient had sleep apnea.

The patient was informed about the risks of the anestheticsurgical procedure in the presence of obstructive sleep apnea and about the need for a pneumology evaluation for maximum safety of the procedure. The case was discussed with the surgeon.

One week after the first visit, the patient returned to the clinic stating he was extremely pleased with the previous visit and that, after another visit with his ENT - who told him of the possibility of the surgery not being completely curative, decided to postpone the procedure until reduction of his body weight and follow-up by the sleep clinic.

\section{DISCUSSION}

Obstructive sleep apnea (OSA) represents an important perioperative challenge for anesthesiologists. This disorder, in which the patient has periods of apnea and hyperpnea during sleep due to complete or partial obstruction of the airways, is associated with episodes of hypoxemia and hypercarbia. Both anesthesia and surgery affect sleep pattern in general. Because obesity is related with sleep apnea, the American Society of Anesthesiology (ASA) recommended recently that obese patients in preoperative appointments should be evaluated for the risk of OSA 9 . This entity increases the risk of cardiac dysrhythmias, myocardial infarction, stroke, and sudden death. It is diagnosed by polysomnography performed at a specialized laboratory. As this implies costs and resources that oftentimes patients do not have, there are several questionnaires based on history, physical examination, and laboratory evaluation whose objective is a more accessible preoperative diagnosis to anesthesiologists in general. Models have been developed and tested in several centers ${ }^{10,11}$.
One of the best questionnaires developed (STOP-BANG) 10 takes into consideration the predisposing factors for OSA, presenting questions easy to approach in an outpatient preanesthetic setting that should be asked routinely.

Patients are considered at high risk for sleep apnea when they answer yes to more than three questions. This test has elevated sensitivity and specificity ${ }^{10}$.

The ASA developed a task-force to evaluate patients at risk for OSA, presenting a 16-item questionnaire divided into physical characteristics, symptoms, and complaints.

STOP-BANG Questionnaire

S - Snoring: Do you snore loudly?

T - Tired: Do you feel tired, sleepy during daytime?

O - Observed: Has anyone observed you stop breathing during sleep?

P - Blood Pressure: Are you being treated or had you been treated for hypertension?

$\mathrm{B}$ - BMI: Body mass index $>35$

A - Age: Age over 50 years

$\mathrm{N}$ - Neck: Neck circumference greater than $40 \mathrm{~cm}$

G - Gender: Male gender

\begin{tabular}{|l} 
ASA Questionnaire \\
I - PHYSICAL CHARACTERISTICS \\
BMI > 35 \\
Neck circumference $>43 \mathrm{~cm}$ \\
Craniofacial abnormalities that affect the airways \\
Anatomical nasal obstruction \\
Hypertrophied tonsils that touch in the middle of the airways \\
2 - SYMPTOMS (presence of two or more symptoms) \\
Frequent snoring \\
Respiratory arrest during sleep \\
Frequent awakening \\
3 - SOMNOLENCE \\
Fatigue or somnolence despite adequate sleep \\
Sleeps easily whenever in calm environments without stimulation \\
Sleepy, aggressive, or distracted child \\
Child difficult to awake
\end{tabular}

Scoring: If two or more items in category 1 are positive, then category 1 is positive. If two or more items in category 2 are positive, then category 2 is positive. If one or more items in category 3 are positive, then category 3 is positive. A patient is at high risk for OSA when two or more categories are positive; or at low risk when only one category is positive. 
The patient presented here had a score of four in the STOP-BANG and high risk in the ASA questionnaire. In his case, he had already been diagnosed by polysomnography, corroborating the importance of these tests when evaluating a patient in the pre-anesthetic outpatient clinic where, oftentimes, patients do not have the time, resources, or access to polysomnography.

\section{Perioperative risks of obstructive sleep apnea}

The risk of anesthesia in this class of patient depends on the severity of OSA and complexity of the surgical-anesthetic procedure. Anesthesia requiring higher dose of opioids, larger surgeries, and those on the airways are considered high risk. Depressor effects of inhalational anesthetics and opioids on the respiratory system cause relaxation of airways musculature, depression of the respiratory center, and deficiency of the compensatory mechanisms by changes in neural function ${ }^{12}$. Complications such as hypoxemia, hypercapnia, cardiac dysrhythmias, myocardial ischemia, impaired airway management, cardiac arrest, and increased rate of postoperative infection have been reported ${ }^{13}$. An increase in postoperative complications, late discharge from the recovery room, with higher incidence of episodes of late desaturation, greater need of admission to the Intensive Care Unit, and increased length of hospitalization are also observed ${ }^{14,15}$.

The difficulty to intubate this group of patients is up to eightfold more frequent than the control group ${ }^{16}$. Thick and short neck, limitation of cervical extension, reduction of thyromental distance, reduction of the caliber of the airways due to the excess of lose tissue, and increased neck circumference are common. Obstructive sleep apnea is a risk factor for difficult airway, impossible airway, and difficulty to ventilate with a face mask. During anesthetic induction the patient should be placed in a comfortable position with elevation of the thorax and head to reduce the closing pressure in the pharynx, increase lung volume, and facilitate direct laryngoscopy. Three-minute preoxygenation with well-adapted face mask prolongs tolerance to apnea, and use of positive pressure in the airways during induction improves oxygenation and prevents airway obstruction. One should think of rapid sequence intubation in obese patients due to the risk of aspiration, remembering that the risks of rapid sequence intubation and desaturation should be weighed against its benefits. Intubation with the patient awake is another possibility, which should be considered when jaw thrust maneuvers, neck extension and mouth opening are not sufficient to maintain the airway patent ${ }^{17}$.

Depression of the activity of airways muscles is dose-dependent, and anesthetics and narcotics affect respiration by chemical and metabolic effects. Whenever possible, the use of these drugs should be avoided ${ }^{18}$. A recent study demonstrated that a fall in arterial blood oxygen saturation in patients on remifentanil was due to a central effect of the drug and not to obstructive apnea. A reduction in rapid eye movement (REM) sleep was also observed in these patients, while awakening during respiratory events increased. Interestingly, a rapid reduction in obstructive apnea episodes with an increase in the number of central apnea was observed ${ }^{12}$.

In the extubation period, one should use the head up or lateral position to maintain the airways and improve oxygenation. Supine position should be avoided. A residual effect of inhalational anesthetic and neuromuscular blocker is harmful. The effects of neuromuscular blockers should be completely reversed at the end of the procedure. Complete awakening and spontaneous respiration should be present before extubation. The use of beta blockers or anti-hypertensive agents may be required at this moment. Thoracoabdominal swing immediately after extubation indicates airways obstruction, which should be treated to avoid pulmonary edema secondary to negative pressure ${ }^{17}$.

In peripheral procedures, blockade is preferable to general anesthesia. General anesthesia is safer than deep sedation and, in patients undergoing airway procedures (for example, bronchoscopy and endoscopy) general anesthesia with safe airways is preferable. Capnography monitoring should be used for sedation of these patients ${ }^{9}$.

Postoperative analgesia should be performed with local anesthetics to avoid adverse effects of opioids. The use of non-steroidal anti-inflammatories, whenever there are no contraindications, is a good choice in this group of patients. In cases opioids cannot be avoided, oximetry and respiratory rate monitoring for 24 hours is mandatory, frequently in the ICU ${ }^{19}$. The route of administration of opioids is not relevant regarding postoperative complications. The head up or lateral position should be maintained in the postoperative period, and it is frequently necessary the use of continuous positive airway pressure (CPAP) or oxygen ${ }^{17}$. Patients already on CPAP should be encouraged to take it to the operating room to be used in the postoperative period.

The ASA recommends that every patient suspected of having OSA should undergo preoperative assessment. This procedure allows adequate approach and anesthetic-surgical planning. Due to the high prevalence of non-diagnosed OSA (more than $24 \%$ of cases), every obese patient should be suspected of having OSA ${ }^{17}$. These patients are at high risk for sedatives and opioids, and they may present severe respiratory events evolving to cardiac arrest. The use of the STOP-BANG and ASA questionnaires in the outpatient clinic or pre-anesthetic rounds is done to stratify patient risk, reducing the possibility of adverse events in patients with the greatest chances of OSA, recognizing it is impossible to eliminate perioperative morbidity and mortality in these patients. Prior assessment favors adequate anesthetic planning, postoperative monitoring, and identification of patients who really would benefit from evaluation with polysomnography and formal OSA treatment ${ }^{20,21}$. 